\title{
The Effects of Patients' and Care-Givers' Knowledge, Attitude, \& Practice (KAP) on Quality of Life Among Thalassemia Major Patients' in Damascus-Syrian Arab Republic
}

\author{
Yaser Adnan Abo Jeesh, PhD Candidate
}

Faculty of Medicine, University of Gezira - Sudan

Magda El-hadi Ahmad Yousif, PhD

Professor, Chairperson of Primary Health Care and Health Education Center, Faculty of Medicine, University of Gezira - Sudan

Moauya Al-Balal Al-Haboub, PhD

Professor of Faculty of Medicine, University of Gezira - Sudan

Doi: 10.19044/esj.2018.v14n12p308 URL:http://dx.doi.org/10.19044/esj.2018.v14n12p308

\begin{abstract}
Background: Thalassemia is the most common autosomal abnormality in Syria. Its complications have an important effect on education; time off school; sport; difference from friends/ siblings; social interactions; and stigmatization. Knowledge of factors associated with quality of life in thalassemia patients is necessary for forming appropriate clinical programs, social support, and improving treatment outcomes. Purpose: The study was to assess the effects of Patients' and Care-givers' Knowledge, Attitude, \& Practice (KAP) with Quality of Life among Thalassemia Major Patients' in Syria. Methods: Cross-sectional descriptive analytical design was conducted at the national thalassemia center in Damascus. WHOQOL-BREF and a questionnaire developed by the researcher were used to measure the participants' knowledge, attitudes and practices of thalassemia. Results: Total of 238 thalassemia patients participated in the study. A statistical significance was found regarding improvement of skill and knowledge scores among caregivers and thalassemia patients before and after receiving the teaching guide from $12.52 \pm 1.77$ to $14.07 \pm 1.01, \mathrm{t}=11.447, \mathrm{p}=0.000$ and from $34.12 \pm 4.50$ to $37.43 \pm 4.61, \mathrm{t}=-8.58, \mathrm{p}=.000$ respectively. Stigmatization was significantly noticed among families caring for thalassemic patients. There were a significant differences in the mean score regarding nutritional status before and after teaching guide paired $t$ test $=12.11, p=0.000$. A statistical significance was found in females regarding social domain $p=0.04$. However, mean scores for overall quality of life were better in females rather than males,
\end{abstract}


but these mean scores were statistically insignificant $p>0.05$. A statistical significant difference in ferritin levels and patient's age was found. Results also revealed that no statistical significant differences was observed between overall quality of life of the four domains in relation to the two treatment groups (subcutaneous vs oral) and age groups. Conclusion: Thalassemia is a socio-economic problem. The most efficient way to reduce risks of having affected patients is by increasing the knowledge through training of parents regarding the disease. Our findings highlighted that there was lack in patients' knowledge and skills regarding the disease, but a remarkable improvement in both knowledge and performance was found after patients received the teaching guide, which lead to an increase in overall patient's quality of life.

Keywords: Thalassemia, Quality of Life, Knowledge

\section{Introduction}

The term Thalassemia was first used by Wipple and Bradford in 1932, derived from Greek word "thlassa" meaning sea and "haemia" meaning blood. (Nagaraj, Umashree et al. 2011). Thalassemia is one of the most common genetic blood disorders in the world, the most severe form is betathalassemia major, which warrants regular blood transfusions at an early age, if it not treated, the result can be early death (El Dakhakhny, Hesham et al. 2011, Safizadeh and Farahmandinia 2012, Elalfy, Farid et al. 2014). As other countries of the Mediterranean region, thalassemia is the most common autosomal disorders of the Syrian people, the number of the total registered thalassemia patients was 8300 in year 2013 and increasing by almost 800 each year, the average carrier rate is $5-7 \%$ of the total population (Al-Zir 2009, Touma, AlKeba et al. 2013). Regular blood transfusions and iron chelation therapy (ICT) have significantly improved survival, reduced morbidity and enhanced health related quality of life (HRQoL) of patients with betathalassemia major (BTM) (Touma, AlKeba et al. 2013, Hatami and Motamed 2014).

There has been very limited researches about the effects of thalassemia on QoL especially for pediatric patients (Hatami and Motamed 2014). No studies have been done to measure or to assess awareness of the Syrian people or families with thalassemic patients regarding knowledge, attitude and practices related to quality of life for these patients in Syria. As such more attention is needed in issues related to knowledge and understanding of the illness, lifelong compliance and adherence to chronic treatment regime and attitudes of patients and family members as well as quality of interpersonal relationships (Jantan, Muhammad et al. 2011).

The most effective way to decrease complications of thalassemia patients is by increasing knowledge, attitude and practice awareness of these 
patients and their parents about the disease and its management, which will result in a positive impact in their quality of life. This will improve their performance to deal with their lifelong disease and its challenges during the period of management (Kourorian Z et al 2014 (Dehkordi and Heydarnejad 2008). The obvious role of parents' education in the prevention of betathalassemia is evident in the study of Dehkordi and Heydarnejad 2008 which indicated that population with poor pre marriage knowledge of the couples about the disease resulted in $62.5 \%$ of the affected children. This problem could have been reduced by improvement of the parents' health education (Dehkordi and Heydarnejad 2008).

In Syria, only one thalassemia organization is found in the capital Damascus that provides the social support and information for the patients and their family. Currently the Syrian Ministry of Health (SMOH) has started with a training program for health personnel to provide good quality care and counselling for the patients and families (Touma, AlKeba et al. 2013). Unfortunately, till now there are no professional psychologists who are working in institutions caring for thalassemia to support and follow up the cases.

Patients with $\beta$-thalassemia major require iron-chelation therapy to avoid the complications of iron overload such as severe anemia with an enlarged spleen, pallor, irritability, growth retardation, delayed puberty, abdominal swelling, and jaundice (Muncie Jr and Campbell 2009, Milat, Wong et al. 2012). Other severe complications such as heart failure, cardiac arrhythmia, liver disease, endocrine complications and infections are common among patients with $\beta$-thalassemia and may impact negatively on patients' quality of life (QOL) (Borgna-Pignatti and Gamberini 2011, Liem, Gilgour et al. 2011, Pennell, Carpenter et al. 2011, Ali, Tarawah et al. 2015).

Effective treatment of patients with $\beta$-thalassemia major consists of regular blood transfusions, intensive administration of iron-chelating agents such as deferoxamine (DFO) by subcutaneous infusions, or deferiprone (DFP) and deferasirox (DFX) that are taken orally, Spleenectomy and Bone marrow transplantation (Schrier and Angelucci 2005, Cohen 2006, Capellini, Cohen et al. 2008, Cianciulli 2008, Vichinsky, Levine et al. 2008, Murad, Moassas et al. 2014). Deferoxamine (Desferal), subcutaneously or intravenously, has been the treatment of choice (Muncie Jr and Campbell 2009). Chelation therapy has two main forms: subcutaneous slow infusion by a portable pump (deferoxamine) and oral (deferasirox, deferiprone), whereas most patients in Syria use classical chelation therapy using desferrioxamine and the infusion pump (Al-Zir 2009, Trachtenberg, Mednick et al. 2012). The cost of treatment per $\beta$-thalassemia patient is up to $\$ 1500.00$ per year (Murad, Moassas et al. 2014).

Serum ferritin is increasing as an indicative of compliance with 
deferasirox treatment (Milat, Wong et al. 2012). Serum ferritin is the best test to screen for iron deficiency anemia (Muncie Jr and Campbell 2009). Consequently, compliance with regular administration of chelation agents is a key feature to the long-term health of thalassaemic patients (Atkin and Ahmad 2000):whereas, the serum ferritin level that identifies safe levels of body iron with respect to the development of complications of iron overload is $<2500$ mcg/L (Olivieri, Nathan et al. 1994, Muncie Jr and Campbell 2009). In a study done by (Pakbaz, Fischer et al. 2005) to investigate the factors that can improve adherence to iron chelation therapy with DFO in patients with thalassemia. Ninety patients with thalassemia major were assessed for adherence to chelation therapy by using Liver Iron Concentration (LIC) and Serum Ferritin (SF). Results showed that patient education and counseling about the benefits of chelation therapy can play an important role in improving adherence. Frequent measurement of liver iron concentration can be valuable in modifying patients' belief about the medication because it can give evidence for the effectiveness of DFO in reducing LIC and therefore lead to a better compliance. (Pakbaz, Fischer et al. 2005).

The magnitude of thalassemia is masked by infections and nutritional deficiencies (Nagaraj, Umashree et al. 2011). Therefore, the thalassemia patients should avoid food rich in iron and drink a cup of tea with every meal because it interferes with iron absorption in the diet patient should also avoid food rich of vitamin- C during meals (Wonke 2001, Caro, Ward et al. 2002, Capellini, Cohen et al. 2008).

The Quality of Life (QoL) of individuals with thalassaemia major is influenced by the following factors: impact of the diagnosis and treatment on family stability and family dynamics, having a chronic condition manifisting by bone deformities, short stature, leading to poor self-image; treatment measures like frequent hospital visits for transfusion; nightly subcutaneous infusions; delayed or absent sexual development and impaired fertility; complications of the disease and therapeutic interventions such as heart disease, bone disease, diabetes, infections; and uncertainties about the future, difficulties in long-term planning (Telfer, Constantinidou et al. 2005, Shaligram, Girimaji et al. 2007, Jafari, Lahsaeizadeh et al. 2008, Scalone, Mantovani et al. 2008). Hence, the impact of iron chelation therapy (ICT) on Quality of Life (QoL) will not be noticed until major complications begin to occur in the teens or early twenties for patients with thalassaemia (Abetz, Baladi et al. 2006, Ansari, Baghersalimi et al. 2014). The aim of this study was to assess the effects of patients' and care-givers' knowledge, attitude, \& practice (KAP) on quality of life among $\beta$-thalassemia major patients in Syria, and according to the research results, we are looking to develop a teaching guide based on the previous assessment of home caregivers and patients regarding to their needs. 


\section{Methods and design \\ Sample and setting}

A cross-sectional descriptive analytical study was performed from 20 September 2016 to 22 December 2016, a total of 238 thalassemia patients and their caregivers who visited the national thalassemia center in Damascus were included in this study. The center was established in 1997 with over 1200 thalassemia patients. The diagnosis of thalassemia major is confirmed using compete blood count and hemoglobin electrophoresis in the center. The center provides blood transfusions, desferrioxamine and social support (Gharaibeh, Amarneh et al. 2009). This site was selected for the purpose of this study, because it is considered the main center that provides a holistic care for thalassemia patients' children/ adolescents or adults in Syria.

The inclusion criteria were, A: (1) All the subjects to be diagnosed as beta-thalassemia major and confirmed in medical record by physician, (2) Those receiving blood transfusion every 3-4 weeks (3) Those taking iron chelation therapy (Desferal medication subcutaneously by using infusion pump at home (4) All patients have to be within the three age categories I) aged 5- to less than 12 years, II) aged 12-19 years, and III) aged =>19 years. B: All caregivers of patients at home (next of kin) were included, one for each patient. An official letter issued from University of Gezira and Ministry of Health in Syria to obtain approval agreement to conduct the study, a permission to use WHOQOL-BREF was taken, also a written consent was obtained from each patient. Home caregivers and patients were assured that there were no harms, risks and discomfort caused to them through participation. The teaching guide was distributed to the patients or caregivers through a period of data collection for initial assessment. Every patient and caregiver was interviewed for an average of one hour to fill the data collection formats directed towards each. All interviews were done with a patient or home caregiver accompanying the child who was attending the center according to his/her appointment for receiving blood transfusion and treatment in the governmental center. The researcher assessed the patients and their caregiver's knowledge and skills in the first interview, followed by handing of the thalassemia guide. Two months later, a second interview was conducted to reassess the participants.

\section{Instrument}

Five tools were used in the process of data collection.

1. Validated World Health Organization Quality of Life (WHOQOLBREF) questionnaire was used for the purpose of this study. The WHOQOLBREF questionnaire covered four domains - physical, psychological, social relationships and environment.

The researcher designed and developed the following structured 
questionnaire:

2. Home caregivers' knowledge about thalassemia major patient interview.

3. Home caregiver attitudes interview sheet.

4. Physical assessment checklist.

5. Designed observational checklist was used to observe patients or their caregivers at their homes while preparing and administering the Desferal subcutaneously.

Demographic characteristics, disease features and complications were analysed with mean score of QOL using chi-square, ANOVA and t-test where appropriate. Statistical significant was considered as P-value $<0.05$. The SPSS version 20 was used to analyse the data.

\section{Results}

Table 1- represents the general characteristics of patients in relation to birth order, gender, educational level, marital status and age groups. Male constituted $53.4 \%$, while $46.6 \%$ were female. As regard age group, two thirds of the sample were childhood and adolescent (72.3\%). 36.6\% were in the age group of 5 to less than 12 years ( $20.6 \%$ male, and $16.0 \%$ female), while $35.7 \%$ were in the age group 12 to less than 19 years $(18.5 \%$ male, and $17.2 \%$ female. Adulthood category for 19 years and above was the lowest about $27.7 \%$ ( $14.3 \%$ of them male and $13.4 \%$ were female). The majority of patients $(71 \%)$ were in school education. 
Table 1: General Characteristics of the patients.

\begin{tabular}{|c|c|c|c|c|c|c|}
\hline \multirow[b]{2}{*}{ Characteristics } & \multicolumn{2}{|l|}{ Male } & \multicolumn{2}{|l|}{ Female } & \multicolumn{2}{|l|}{ Total } \\
\hline & $\mathrm{No}=127$ & $\%$ & $\mathrm{No}=111$ & $\%$ & $\mathrm{No}=238$ & $\%$ \\
\hline Patient gender & 127 & 53.4 & 111 & 46.6 & 238 & 100 \\
\hline \multicolumn{7}{|l|}{$\begin{array}{l}\text { Birth order of thalassemia } \\
\text { Patient in the family: }\end{array}$} \\
\hline First & 56 & 23.5 & 41 & 17.2 & 97 & 40.8 \\
\hline Second & 38 & 16 & 30 & 12.6 & 68 & 28.6 \\
\hline Third & 20 & 8.4 & 18 & 7.6 & 38 & 16 \\
\hline Fourth & 10 & 4.2 & 12 & 5 & 22 & 9.2 \\
\hline Fifth & 2 & 0.8 & 7 & 2.9 & 9 & 3.8 \\
\hline Sixth and More & 1 & 0.4 & 3 & 1.3 & 4 & 1.7 \\
\hline $\begin{array}{l}\text { Educational level: } \\
\text { KG } \\
\text { Elementary } \\
\text { Preparatory } \\
\text { Secondary } \\
\text { Diploma } \\
\text { University }\end{array}$ & $\begin{array}{l}8 \\
40 \\
25 \\
30 \\
7 \\
17\end{array}$ & \begin{tabular}{l|l}
3.4 \\
16.8 \\
10.5 \\
12.6 \\
2.9 \\
7.1
\end{tabular} & $\begin{array}{l}9 \\
25 \\
28 \\
21 \\
7 \\
21\end{array}$ & $\begin{array}{l}3.8 \\
10.5 \\
11.8 \\
8.8 \\
2.9 \\
8.8 \\
\end{array}$ & $\begin{array}{l}17 \\
65 \\
53 \\
51 \\
14 \\
38\end{array}$ & $\begin{array}{c}7.1 \\
27.3 \\
22.3 \\
21.4 \\
5.9 \\
16 \\
\end{array}$ \\
\hline $\begin{array}{l}\text { Marital Status: } \\
\text { Single } \\
\text { Married } \\
\text { Not applicable* }\end{array}$ & $\begin{array}{l}52 \\
8 \\
67\end{array}$ & $\begin{array}{l}21.8 \\
3.4 \\
28.2\end{array}$ & $\begin{array}{l}52 \\
5 \\
54\end{array}$ & $\begin{array}{l}21.8 \\
2.1 \\
22.7\end{array}$ & $\begin{array}{l}104 \\
13 \\
121\end{array}$ & $\begin{array}{c}43.7 \\
5.5 \\
50.8\end{array}$ \\
\hline \multicolumn{7}{|l|}{ Age groups: } \\
\hline \multirow{3}{*}{$\begin{array}{l}\text { Childhood ( } 5 \text { - less than } 12 \text { years) } \\
\text { Adolescent ( } 12-\text { less than } 19 \text { years) } \\
\text { Adulthood ( } 19 \text { years \& above) }\end{array}$} & 49 & 20.6 & 38 & 16.0 & 87 & 36.6 \\
\hline & 44 & 18.5 & 41 & 17.2 & 85 & 35.7 \\
\hline & 34 & 14.3 & 32 & 13.4 & 66 & 27.7 \\
\hline Mean + SD & & & & & $15.67+$ & \\
\hline
\end{tabular}

*Applicable (Patients' age equal or more 16 years)

*Not applicable (Patients' age less than 16 years old)

Table 2 portrays home caregivers' characteristics. Mothers were caregivers for patients in about two thirds of the sample $(62.6 \%)$. Fathers and patient himself were considered caregivers in $8.0 \%, 27.7 \%$ of cases respectively. The majority of caregivers $75.6 \%$ were married. As regard to educational level of caregivers, data indicated that $47.1 \%, 53 \%$ of caregivers got their school education and higher education respectively. $66.4 \%$ of caregivers were un-employed. 
Table 2: Home caregivers' characteristics

\begin{tabular}{lll}
\hline Characteristics & No & \\
$\begin{array}{l}\text { Caregivers: } \\
\text { Mother }\end{array}$ & $\mathrm{N}=238$ & $\%$ \\
$\begin{array}{l}\text { Father } \\
\text { Brother / Sister }\end{array}$ & 149 & 62.6 \\
$\begin{array}{l}\text { Patient himself } \\
\text { Marital Status }\end{array}$ & 19 & 8.0 \\
Single & 66 & 1.7 \\
Married & & 27.7 \\
$\begin{array}{l}\text { Divorced / Widowed } \\
\text { Level of Education }\end{array}$ & 57 & 23.9 \\
$\begin{array}{l}\text { Basic education } \\
\text { Preparatory }\end{array}$ & 180 & 75.6 \\
Secondary & 1 & 0.4 \\
$\begin{array}{l}\text { Diploma } \\
\text { University } \\
\text { Occupational status }\end{array}$ & 9 & \\
Governmental sector employee & 31 & 3.8 \\
$\begin{array}{l}\text { Private sector employee } \\
\text { Retired }\end{array}$ & 80 & 9.7 \\
Un-employed & 67 & 33.6 \\
Other & 59 & 28.2 \\
& 16 & 24.8 \\
\hline
\end{tabular}

Table 3 portrays about two thirds of sample $(66.0 \%)$ had insufficient monthly income at all while $29.8 \%$ of families had sufficient income. More than half of the sample $(58.4 \%)$ was from rural areas. Also the table depicts great proportion of sample $(65.5 \%)$ had consanguineous parents. Less than half of sample $(44.5 \%)$ had a positive family history of thalassemia. 
Table 3. Percent distribution of socioeconomic status and family history

\begin{tabular}{|c|c|c|}
\hline \multicolumn{3}{|l|}{ Data } \\
\hline Categorical monthly income & & \\
\hline in Syrian Pound (SP) & 71 & 29.8 \\
\hline Sufficient & 10 & 4.2 \\
\hline Sufficient and few saved & 157 & 66.0 \\
\hline \multicolumn{3}{|l|}{ Insufficient at all } \\
\hline Type of accommodation (before War) & 185 & 77.7 \\
\hline Own & 53 & 22.3 \\
\hline \multicolumn{3}{|l|}{ Rent } \\
\hline Type of accommodation (During War) & 79 & 33.7 \\
\hline Own & 72 & 30.0 \\
\hline Rent & 87 & 36.3 \\
\hline \multicolumn{3}{|l|}{ Inner Refugee } \\
\hline Residence & 99 & 41.6 \\
\hline Urban & 139 & 58.4 \\
\hline \multicolumn{3}{|l|}{ Rural } \\
\hline Mean Crowding index & \multicolumn{2}{|c|}{$.6187+.2814$} \\
\hline \multicolumn{3}{|l|}{ Parents' relatives (consanguinity): } \\
\hline Yes & 156 & 65.5 \\
\hline No & 82 & 34.5 \\
\hline Degree of relatives: & $\mathbf{N}=\mathbf{1 5 0}$ & \\
\hline Daughter of cousin/uncle & 70 & 46.7 \\
\hline Daughter of aunt & 48 & 32.0 \\
\hline Other & 32 & 21.3 \\
\hline Anyone affected with thalassaemia & $\mathbf{N}=\mathbf{2 3 8}$ & \\
\hline Within the whole family: & 106 & 44.5 \\
\hline Yes & 132 & 55.5 \\
\hline No & $\mathbf{N}=109$ & \\
\hline Degree of relative: & 50 & 45.9 \\
\hline First class & 39 & 35.8 \\
\hline (Father, mother, brother, sister) & 20 & 18.3 \\
\hline \multicolumn{3}{|l|}{ Second class } \\
\hline (Grandfather/mother, cousins/aunt, uncle & $\mathbf{N}=109$ & \\
\hline Third class & 10 & 9.2 \\
\hline \multirow[t]{2}{*}{ (Others from the family) } & 6 & 5.5 \\
\hline & 91 & 83.5 \\
\hline Type of thalassaemia: & 2 & 1.8 \\
\hline Minor & & \\
\hline Intermediate & & \\
\hline Major & & \\
\hline I don't know & & \\
\hline
\end{tabular}

Table 4 Depicts great proportion of sample $(76.9 \%)$ had knowledge about thalassemia. Nearly third of sample $37.0 \%$ knew the causes of thalassemia and $16.6 \%$ knew the methods of prevention. Regarding the source of information the doctors were the main source for the majority of the sample $60.0 \%$. 
Table 4: Home caregivers' knowledge about Thalassemia

\begin{tabular}{|l|l|l|}
\hline Data & No. & $\%$ \\
Knowledge about thalassaemia: & $\mathrm{N}=238$ & \\
No & 183 & 76.9 \\
Areas of information: & 55 & 23.1 \\
Causes & $\mathrm{N}=185$ & \\
Prevention & 67 & 37.0 \\
Signs \& symptoms & 30 & 16.6 \\
Modes of transmission & 31 & 17.1 \\
Treatment & 19 & 10.5 \\
Complications & 32 & 17.7 \\
Sources of information: & 2 & 1.1 \\
Doctors & $\mathrm{N}=185$ & \\
Nurse & 111 & 60.0 \\
Family & 45 & 24.3 \\
Books & 23 & 12.4 \\
Brochures \& Newspaper & 3 & 1.6 \\
& 3 & 1.6 \\
\hline
\end{tabular}

Table 5 shows an improvement of caregivers' knowledge after receiving the teaching guide in blood components \& functions, hemoglobin level, causes of thalassemia, prevention, treatment and nutrition. There is an improvement in the overall caregivers' knowledge regarding thalassemia, whereas the mean and standard deviation was $34.12 \pm 4.50$ before receiving the teaching guide and $37.43 \pm 4.61$ after receiving the teaching guide.

Table 5: Mean score caregivers' knowledge about Thalassemia of pre and post teaching guide

\begin{tabular}{|l|l|l|l|l|}
\hline Area of knowledge & Mean score & \multicolumn{2}{|l|}{} \\
\hline & $\begin{array}{l}\text { Before Receiving } \\
\text { Guide }\end{array}$ & $\begin{array}{l}\text { After Receiving } \\
\text { Guide }\end{array}$ & Paired t. test & $\begin{array}{l}\text { P. } \\
\text { value }\end{array}$ \\
\hline 1. Blood components \& functions & 4.07 & 5.30 & -19.97 & $.000^{*}$ \\
\hline 2. Hemoglobin level & 1.80 & 2.15 & -5.65 & $.000^{*}$ \\
\hline 3. Causes of thalassaemia & 7.29 & 8.65 & -11.03 & $.000^{*}$ \\
\hline 4. Disease transmission & 2.90 & 2.97 & -0.719 & 0.473 \\
\hline 5. Prevention & 3.12 & 3.49 & -6.12 & $.000^{*}$ \\
\hline 6. Treatment & 13.81 & 13.04 & 3.77 & $.000^{*}$ \\
\hline 7. Nutrition & 1.72 & 1.11 & 12.11 & $.000^{*}$ \\
\hline Overall knowledge & $34.12 \pm 4.50$ & $37.43 \pm 4.61$ & -8.58 & $.000^{*}$ \\
\hline & $*$ Significant $\mathrm{P}<0.05$ & & \\
\hline
\end{tabular}

Table 6 Depicts great proportion of sample (59.2\%) had no difference from peers. Nearly one third of the sample $37.1 \%$ had physical changes such as growth retardation, $24.7 \%$ had facial changes, $16.5 \%$ had a weakness \& exercise intolerance, $11.3 \%$ had skin color changes and $10.3 \%$ had abdominal changes and the majority $67.7 \%$ were upset from these differences. Regarding to social activities, more than half of the sample $55.9 \%$ didn't engage in group activities and play. 
Table 6: Description of Self -Image of Patients with Thalassaemia Major

\begin{tabular}{|c|c|c|c|c|c|c|}
\hline \multirow[b]{2}{*}{ Variables } & \multicolumn{2}{|l|}{ Male } & \multicolumn{2}{|l|}{ Female } & \multicolumn{2}{|l|}{ Total } \\
\hline & $\mathrm{No}=127$ & $\%$ & $\mathrm{No}=111$ & $\%$ & $\mathrm{No}=238$ & $\%$ \\
\hline $\begin{array}{l}\text { Different from peers: } \\
\text { Yes } \\
\text { No }\end{array}$ & $\begin{array}{l}52 \\
75\end{array}$ & $\begin{array}{l}21.8 \\
31.5\end{array}$ & $\begin{array}{l}45 \\
66\end{array}$ & $\begin{array}{l}18.9 \\
27.7\end{array}$ & $\begin{array}{l}97 \\
141\end{array}$ & $\begin{array}{l}40.8 \\
59.2\end{array}$ \\
\hline Total & No. 52 & $\%$ & No. 45 & $\%$ & No. 97 & $\%$ \\
\hline $\begin{array}{l}\text { Different in what: } \\
\text { Facial changes } \\
\text { Physical changes as } \\
\text { growth retardation } \\
\text { Weakness \& exercise } \\
\text { intolerance } \\
\text { Skin color changes } \\
\text { Abdominal enlargement }\end{array}$ & $\begin{array}{l}13 \\
20 \\
10 \\
7 \\
2\end{array}$ & $\begin{array}{l}13.4 \\
20.6 \\
10.3 \\
7.2 \\
2.1\end{array}$ & $\begin{array}{l}11 \\
16 \\
6 \\
4 \\
8\end{array}$ & $\begin{array}{l}11.3 \\
16.5 \\
6.2 \\
4.1 \\
8.2\end{array}$ & $\begin{array}{l}24 \\
36 \\
16 \\
11 \\
10\end{array}$ & $\begin{array}{r}24.7 \\
37.1 \\
16.5 \\
11.3 \\
10.3\end{array}$ \\
\hline Total & No. 51 & $\%$ & No. 42 & $\%$ & No. 93 & $\%$ \\
\hline $\begin{array}{l}\text { Disturbed from these } \\
\text { differences: } \\
\text { Yes } \\
\text { No }\end{array}$ & $\begin{array}{l}34 \\
17\end{array}$ & $\begin{array}{l}36.6 \\
18.3\end{array}$ & $\begin{array}{l}29 \\
13\end{array}$ & $\begin{array}{l}31.2 \\
14\end{array}$ & $\begin{array}{l}63 \\
30\end{array}$ & $\begin{array}{l}67.7 \\
32.3\end{array}$ \\
\hline Total & No. 127 & $\%$ & No. 111 & $\%$ & No. & $\%$ \\
\hline $\begin{array}{l}\text { Have special interest and } \\
\text { hobbies } \\
\text { Yes } \\
\text { No }\end{array}$ & $\begin{array}{l}110 \\
17 \\
\end{array}$ & $\begin{array}{l}46.2 \\
7.1 \\
\end{array}$ & $\begin{array}{l}91 \\
20 \\
\end{array}$ & $\begin{array}{l}38.2 \\
8.4\end{array}$ & $\begin{array}{l}201 \\
37 \\
\end{array}$ & $\begin{array}{l}84.5 \\
15.5\end{array}$ \\
\hline Total & No. 127 & $\%$ & No. 111 & $\%$ & No. & $\%$ \\
\hline $\begin{array}{l}\text { Engage in group activities } \\
\text { and play } \\
\text { Yes } \\
\text { No }\end{array}$ & $\begin{array}{l}53 \\
74\end{array}$ & $\begin{array}{l}22.3 \\
31.1\end{array}$ & $\begin{array}{l}52 \\
59\end{array}$ & $\begin{array}{l}21.8 \\
24.8\end{array}$ & $\begin{array}{l}105 \\
133\end{array}$ & $\begin{array}{l}44.1 \\
55.9\end{array}$ \\
\hline $\begin{array}{l}\text { Reasons for not engaging } \\
\text { in activities } \\
\text { Shamed face } \\
\text { Inapproperate physical } \\
\text { fitness } \\
\text { Others } \\
\text { Total }\end{array}$ & $\begin{array}{l}12 \\
32 \\
29 \\
73\end{array}$ & $\begin{array}{l}9.2 \\
24.6 \\
22.3 \\
56.2\end{array}$ & $\begin{array}{l}18 \\
20 \\
19 \\
57\end{array}$ & $\begin{array}{l}13.8 \\
15.4 \\
16.4 \\
43.8\end{array}$ & $\begin{array}{l}30 \\
52 \\
48 \\
130\end{array}$ & $\begin{array}{l}23.1 \\
40.0 \\
36.9 \\
100\end{array}$ \\
\hline
\end{tabular}

Table 7 shows $(39.9 \%)$ of sample had compliance with treatment. Great proportion of the sample $(61.7 \%)$ had a capability to inject him/herself with Desferal by using pump. Regarding reasons for not injecting Desferal subcutaneously, third of the sample (32.5\%) answered they had a lack of skills, $(22.5 \%)$ were afraid and $(45.0 \%)$ had been both lacking of skills and afraid. 
Table 7: Shows percent distribution of patients' / caregivers' behaviors regarding Desferal injection

\begin{tabular}{|c|c|c|c|c|c|c|}
\hline \multirow[b]{2}{*}{ Behavior } & \multicolumn{2}{|l|}{ Male } & \multicolumn{2}{|l|}{ Female } & \multirow{2}{*}{$\begin{array}{l}\text { Total } \\
\mathrm{No}=238\end{array}$} & \multirow[b]{2}{*}{$\%$} \\
\hline & $\mathrm{No}=127$ & $\%$ & $\mathrm{No}=111$ & $\%$ & & \\
\hline $\begin{array}{l}\text { Compliance with treatment } \\
\text { Yes } \\
\text { No } \\
\text { Not Apply** } \\
\text { Capability to inject him/herself } \\
\text { with Desferal by using pump } \\
\text { Yes } \\
\text { No } \\
\text { Total } \\
\text { Reasons for not injecting } \\
\text { Desferal Subcutaneously: } \\
\text { A-lack of skills } \\
\text { B - Afraid } \\
\text { C -Both A \& B } \\
\text { Total }\end{array}$ & $\begin{array}{l}40 \\
20 \\
60 \\
7 \\
2 \\
9 \\
18\end{array}$ & $\begin{array}{l}34.8 \\
17.4 \\
52.2 \\
\\
17.5 \\
5.0 \\
22.5 \\
45.5\end{array}$ & $\begin{array}{l}47 \\
8 \\
56 \\
\\
31 \\
24 \\
55 \\
\\
6 \\
7 \\
9 \\
22\end{array}$ & $\begin{array}{l}19.7 \\
3.4 \\
23.5 \\
\\
27.0 \\
20.9 \\
47.8 \\
\\
15.0 \\
17.5 \\
22.5 \\
55.0\end{array}$ & $\begin{array}{l}95 \\
20 \\
123 \\
71 \\
44 \\
115 \\
\\
13 \\
9 \\
18 \\
40\end{array}$ & $\begin{array}{l}39.9 \\
8.4 \\
51.7 \\
\\
61.7 \\
38.3 \\
100 \\
\\
32.5 \\
22.5 \\
45.0 \\
100\end{array}$ \\
\hline
\end{tabular}

**Not apply means that patients whose age less than 15 years old do not use Desferal by using infusion pump.

Table 8 Shows descriptive statistics for serum ferritin and hemoglobin levels $(3852,8.25)$ respectively.

\begin{tabular}{|l|l|l|l|l|l|l|l|}
\hline & $\mathrm{N}$ & Minimum & Maximum & Mean & $\begin{array}{l}\text { Std. } \\
\text { Deviation }\end{array}$ & F & Sig \\
\hline $\begin{array}{l}\text { Serum Ferritin } \\
\text { level }\end{array}$ & 238 & 374.0 & 18575.0 & 3852.0 & 2700.35 & 6.46 & $.002^{*}$ \\
\hline $\begin{array}{l}\text { Hemoglobin } \\
\text { Level }\end{array}$ & 238 & 5.9 & 11.3 & 8.255 & 0.938 & 0.66 & .52 \\
\hline
\end{tabular}

Table 9 shows changes in physical structure of thalassemia patients. Regarding to changes in physical growth retardation findings show half of sample had normal physical growth, while quarter had changes in height and weight. About two thirds of sample had normal facial bone, while (40\%) had different facial bone changes. More than two thirds of sample had normal abdomen, while (28\%) had an enlargement in the abdomen. 
Table 9: Changes in Physical Structure of Thalassaemic patients

\begin{tabular}{l|l|l|} 
Changes in Physical Structure & $\mathrm{N}=238$ & $\%$ \\
Physical growth retardation $(\mathrm{wt}+\mathrm{ht})$ & & \\
Height & 35 & 14.7 \\
Weight & 24 & 10.1 \\
Height and weight & 58 & 24.4 \\
Normal & 121 & 50.8 \\
Facial Bone changes & & \\
Protrusion of the lip and upper central incisors. & & \\
Prominent frontal and parietal bones & 39 & 16.4 \\
Flat or depressed bridge of the nose & 26 & 10.9 \\
Enlarged maxilla & 12 & 5.0 \\
Normal & 19 & 8.0 \\
Abdominal enlargement & 142 & 59.7 \\
Enlarged & & \\
Normal & & \\
& 67 & 28.2 \\
& 171 & 71.8
\end{tabular}

The results show there was an improvement in all steps of this procedure and there was a statistical significant in overall caregivers' and patients' regarding this skill, whereas the mean and standard deviation was $12.52 \pm 1.77$ before receiving the teaching guide and $14.07 \pm 1.01$ after receiving the teaching guide (table 10).

Table 10: Distribution of caregivers or thalassemic patients according to steps of administering Desferal Sc. By using infusion pump

\begin{tabular}{|c|c|c|c|c|}
\hline \multirow[t]{2}{*}{ Steps for administering Desferal SC } & \multicolumn{2}{|c|}{$\begin{array}{l}\text { Before Guide } \\
\mathrm{n}=115\end{array}$} & \multicolumn{2}{|c|}{$\begin{array}{l}\text { After Guide } \\
\mathrm{n}=115\end{array}$} \\
\hline & No $\%$ & Yes $\%$ & No $\%$ & Yes $\%$ \\
\hline 1. Prepare the equipments & 5.2 & 94.8 & 0.0 & 100.0 \\
\hline $\begin{array}{l}\text { 2. Put some pain-killer cream on the skin before the } \\
\text { injection. }\end{array}$ & 93.9 & 6.1 & 79.1 & 20.9 \\
\hline 3. Clean and dry hands before the injection. & 33.9 & 66.1 & 10.4 & 89.6 \\
\hline $\begin{array}{l}\text { 4. Sterilize and clean the injection bottle with alcoholic } \\
\text { swab. }\end{array}$ & 53.0 & 47.0 & 16.5 & 83.5 \\
\hline $\begin{array}{l}\text { 5. Calculate the Desferal dosage according to the } \\
\text { doctor prescription. }\end{array}$ & 3.5 & 96.5 & 1.7 & 98.3 \\
\hline 6. For every bottle, inject $4-5$ c.c. injection water. & 4.3 & 95.7 & 1.7 & 98.3 \\
\hline $\begin{array}{l}\text { 7. Withdraw Desferal as prescribed with a syringe } \\
10 \mathrm{~cm} \text {. }\end{array}$ & 7.8 & 92.2 & 7.8 & 92.2 \\
\hline $\begin{array}{l}\text { 8. Connect the intravenous catheter to syringe and } \\
\text { fixed it to pump. }\end{array}$ & 0.0 & 100.0 & 0.0 & 100.0 \\
\hline 9. Select appropriate injection site & 4.3 & 95.7 & 0.9 & 99.1 \\
\hline $\begin{array}{l}\text { 10. The needle should go all the way beneath the skin } \\
\text { with } 45 \text { angles. }\end{array}$ & 27.0 & 73.0 & 12.2 & 87.8 \\
\hline $\begin{array}{l}\text { 11. Fix the position of the intravenous catheter with } \\
\text { adhesive tape }\end{array}$ & 8.7 & 91.3 & 5.2 & 94.8 \\
\hline 12. Injection speed = length of Desferal occupying the & 24.3 & 75.7 & 9.6 & 90.4 \\
\hline
\end{tabular}




\begin{tabular}{|c|c|c|c|c|}
\hline \multicolumn{5}{|l|}{ syringe / injection time. } \\
\hline $\begin{array}{l}\text { 13. Be sure the pump is working by pressing on the } \\
\text { button of operation. }\end{array}$ & 3.5 & 96.5 & 2.6 & 97.4 \\
\hline $\begin{array}{l}\text { 14. Observe injection site } \& \text { stop the infusion bump } \\
\text { when medicine finished. }\end{array}$ & 8.7 & 91.3 & 6.1 & 93.9 \\
\hline $\begin{array}{l}\text { 15. Clean and replace all equipments after the } \\
\text { injection. }\end{array}$ & 33 & 67 & 13 & 87 \\
\hline 16. Clean and dry hands after the injection. & 38.3 & 61.7 & 25.2 & 74.8 \\
\hline Mean \pm SD & \multicolumn{2}{|c|}{$12.52 \pm 1.77$} & \multicolumn{2}{|c|}{$14.07 \pm 1.01$} \\
\hline t-test ( $p$ value) & \multicolumn{4}{|c|}{$11.447(0.000)^{*}$} \\
\hline
\end{tabular}

*Significant at level $\mathrm{p}<=0.05$

The findings show there were differences between adolescents and adults regarding to the four domains of quality of life, and it was higher in adults than adolescents but without significant relationship (table 11).

Table 11: Relationship between quality of life domains and patients age group

\begin{tabular}{|c|c|c|c|c|c|c|}
\hline \multicolumn{2}{|c|}{ Patient Profile } & \multicolumn{5}{|c|}{ Quality of life Domains } \\
\hline & & Physical & Psychological & Soc & Environmental & Oyerall \\
\hline \multirow{2}{*}{$\begin{array}{l}\text { Patient } \\
\text { age } \\
\text { group } \\
\mathrm{n}=158\end{array}$} & $\begin{array}{l}12- \\
\geq 19 \\
n=88\end{array}$ & $62.4 \pm 16.3$ & $59.8 \pm 19.6$ & $65.5 \pm 26.3$ & $61.5 \pm 19.7$ & $62.3 \pm 18.0$ \\
\hline & $\begin{array}{l}19- \\
\mathrm{n}=70\end{array}$ & $67.2 \pm 18.0$ & $63.8 \pm 20.1$ & $72.4 \pm 21.3$ & $66.6 \pm 16.6$ & $67.5 \pm 16.4$ \\
\hline \multicolumn{2}{|c|}{$\mathrm{t}$ - test ( $\mathrm{p}$ value) } & $\begin{array}{l}1.72 \\
(0.083)\end{array}$ & $\begin{array}{l}0.932 \\
(0.204)\end{array}$ & $\begin{array}{l}1.94 \\
(0.077)\end{array}$ & $\begin{array}{l}1.19 \\
(0.088)\end{array}$ & $\begin{array}{l}1.89 \\
(0.060)\end{array}$ \\
\hline
\end{tabular}

*Significant at level $\mathrm{p}<=0.05$

The findings show there were differences between males and females regarding to the four domains of quality of life, and it were higher in males than females in psychological and environmental domains, while it was statistically significant in social relations in females than males $(71.2 \pm 22.2$ compaed with $66.1 \pm 26.2)(\mathrm{p}=0.04)$, the overall quality of life was higher in females than males but without statistically significant (table 12).

Table 12: Relationship between quality of life domains and the patient gender

\begin{tabular}{|l|l|l|l|l|l|l|}
\hline \multicolumn{2}{|l|}{ Patient profile } & \multicolumn{2}{l|}{ Quality of Life Domains } \\
\cline { 3 - 7 } & $\begin{array}{l}\text { Physical } \\
\text { Health }\end{array}$ & Psychological & $\begin{array}{l}\text { Social } \\
\text { Relationships }\end{array}$ & Environmental & $\begin{array}{l}\text { Overall } \\
\text { Quality of } \\
\text { Life }\end{array}$ \\
\hline $\begin{array}{l}\text { gender } \\
\mathrm{n}=158\end{array}$ & $\begin{array}{l}\text { Male } \\
\mathrm{n}=82\end{array}$ & $64.1 \pm 18.4$ & $62.1 \pm 20.7$ & $66.1 \pm 26.2$ & $64.2 \pm 20.0$ & $64.2 \pm 18.8$ \\
\cline { 2 - 7 } & $\begin{array}{l}\text { Female } \\
\mathrm{n}=76\end{array}$ & $65.0 \pm 15.8$ & $61.0 \pm 19.5$ & $71.2 \pm 22.2$ & $63.2 \pm 16.9$ & $65.1 \pm 15.9$ \\
\hline $\begin{array}{l}\text { t-test } \\
\text { (p value) }\end{array}$ & $\begin{array}{l}0.358 \\
(0.61)\end{array}$ & $\begin{array}{l}0.322 \\
(0.585)\end{array}$ & $\begin{array}{l}1.334 \\
0.04^{*}\end{array}$ & $\begin{array}{l}0.330 \\
(0.089)\end{array}$ & 0.033 \\
\hline
\end{tabular}


The findings show there were differences between the four domains of quality of life regarding two types of treatment (subcutaneous and oral), subcutaneous treatment was higher in all domains, the overall quality of life was higher also in subcutaneous but not statistically significant (table 13).

Table 13: Relationship between quality of life domains and the type of treatment Patient profile Quality of Life Domains

\begin{tabular}{|c|c|c|c|c|c|c|}
\hline & \multirow{3}{*}{$\begin{array}{l}\text { Physical } \\
\text { Health } \\
65.7 \pm 18.2\end{array}$} & \multirow{3}{*}{$\begin{array}{l}\text { Psychological } \\
62.6 \pm 21.3\end{array}$} & \multirow{3}{*}{$\begin{array}{l}\text { Social } \\
\text { Relationships }\end{array}$} & \multirow{3}{*}{$\begin{array}{l}\text { Environmental } \\
64.7 \pm 18.8\end{array}$} & \multirow{3}{*}{$\begin{array}{l}\text { Overall } \\
\text { Quality } \\
\text { of Life } \\
65.2 \pm 17.6\end{array}$} \\
\hline & & & & & & \\
\hline $\begin{array}{l}\text { Type of } \\
\text { treatment }\end{array}$ & $\begin{array}{l}\text { Subcutaneous } \\
\mathrm{n}=115\end{array}$ & & & & & \\
\hline $\mathrm{n}=158$ & $\begin{array}{l}\text { Oral } \\
n=43\end{array}$ & $\begin{array}{l}61.0 \pm \\
14.3\end{array}$ & $59.5 \pm 16.7$ & $65.6 \pm 23.8$ & $62.1 \pm 18.3$ & $62.1 \pm 16.0$ \\
\hline \multicolumn{2}{|c|}{$\begin{array}{l}\text { t-test ( } p \text { value) } \\
\text { ( } p \text { value) }\end{array}$} & $\begin{array}{l}1.59 \\
(0.062)\end{array}$ & $\begin{array}{l}0.073 \\
(0.090)\end{array}$ & $\begin{array}{l}0.683 \\
(0.645)\end{array}$ & $\begin{array}{l}1.06 \\
(0.289)\end{array}$ & $\begin{array}{l}1.147 \\
(0.233)\end{array}$ \\
\hline
\end{tabular}

*Significant at level $\mathrm{p}<=0.05$

Table 14: Relationship of Quality of Life Level with Overall Knowledge, Performance and Attitude of Caregiver

\begin{tabular}{|c|c|c|}
\hline \multirow[t]{2}{*}{ Caregiver knowledge, Performance and Attitude (KAP) } & \multicolumn{2}{|c|}{ Quality of Life Level } \\
\hline & $\begin{array}{l}\text { Unsatisfactory } \\
\text { QoL }\end{array}$ & Satisfactory QoL \\
\hline $\begin{array}{l}\text { Overall knowledge Before guide } \\
\mathrm{n}=238\end{array}$ & $32.72 \pm 3.34$ & $34.96 \pm 4.25$ \\
\hline t-test ( $\mathrm{p}$ value) & \multicolumn{2}{|l|}{$1.77(0.059)$} \\
\hline $\begin{array}{l}\text { Overall knowledge After guide } \\
n=238\end{array}$ & $32.72 \pm 6.51$ & $35.86 \pm 4.76$ \\
\hline t-test ( $p$ value) & \multicolumn{2}{|l|}{$2.53(0.023)^{*}$} \\
\hline Overall Performance Before guide $n=115$ & $9.83 \pm 1.58$ & $12.90 \pm 1.36$ \\
\hline t-test ( $p$ value) & \multicolumn{2}{|l|}{$8.062(0.169)$} \\
\hline $\begin{array}{l}\text { Overall Performance After guide } \\
n=115\end{array}$ & $14.00 \pm 1.41$ & $14.07 \pm 1.01$ \\
\hline t-test ( $\mathrm{p}$ value) & \multicolumn{2}{|l|}{$0.109(0.642)$} \\
\hline Overall Attitude & $62.45 \pm 8.75$ & $60.58 \pm 9.73$ \\
\hline t-test ( $p$ value) $n=158$ & \multicolumn{2}{|l|}{$2.562(0.252)$} \\
\hline
\end{tabular}

\section{Discussion}

Beta-thalassemia major affects males and females equally and no sex difference was reported (Muncie Jr and Campbell 2009, Perisano, Marzetti et al. 2012). Findings of this study show a statistical significant was found in females regarding to social domain $(p=0.04)$. However, mean scores for overall quality of life were better in females rather than males, but these mean scores were statistically insignificant $(\mathrm{p}>0.05)$. These findings were consistent with those of Dahlui and colleagues (Dahlui, Hishamshah et al. 2009) and (Gharaibeh and Gharaibeh 2012) regarding gender, male and female children 
reported similar mean score for quality of life. Furthermore, the findings showed that patient's gender was not associated with HRQoL scores. These findings were inconsistent with those reported by Seyedifar et al (Seyedifar, Dorkoosh et al. 2016) and Ansari et al (Ansari, Baghersalimi et al. 2014) who found that there were significant differences in quality of life according to gender, female patients had better quality of life than males significantly $(\mathrm{p}=0.05)$.

Regarding socio-economic status, results show two thirds of families said their monthly income was insufficient at all time. This finding was congruent with other studies that reported nearly half of the thalassemia families had employment and financial problems as a result of thalassemia, presenting thalassemia as a socio-economic problem (Zani, Di Palma et al. 1995, Aydinok, Erermis et al. 2005, Khurana, Katyal et al. 2006, Gharaibeh and Mater 2009, Ayoub, Radi et al. 2013). A further study carried out by Prasomsuk et al. reported that mothers caring for children with thalassemia major in Thailand had financial difficulty due to loss of income and increased expenses related to treatment, transportation and living costs during their children's admission to the hospital was the main cause of financial burden for these mothers. Not surprisingly, the burden became greater when the children became sick (Prasomsuk, Jetsrisuparp et al. 2007).

In our study, parents are consanguineous in $65.5 \%$ of patients' families. Consanguineous marriage is a social phenomenon among Syrians, it is higher in urban than rural areas $30.3 \%$ and $39.8 \%$ respectively (Othman and Saadat 2009). In the present study, 23.1\% of the sample had knowledge deficit regarding causes, prevention, signs and symptoms, transmission, treatment and complications of thalassemia. Many other studies (Mrayyan, Al-Omary et al. , Hanl, Han et al. 1992, Seyam and Assemi 2010, Miri-Moghaddam, ZadehVakili et al. 2011, Shrivastava, Sinha et al. 2011, Al Hajeri and Al Arrayed 2012, Ishaq, Hasnain Abid et al. 2012, Kourorian, Azarkeivan et al. 2014) reported there was a lack of information and knowledge through the thalassemia patients and families regarding the signs and symptoms, causes and treatment of this disorder.

Health professionals are facing many problems in giving care to thalassemia patients. The main problem is parents' lack of awareness about the disease. Medical staff can help to increase knowledge of families' having patients with thalassemia disorder (Dehkordi and Heydarnejad 2008, Patricia Ault 2009). In this study, caregivers' overall knowledge score ranged from 16 - 45 out of 50 regarding thalassemia major and its management before teaching guide with a mean score $\mathrm{M} \pm \mathrm{SD}(34.12 \pm 4.50)$, while after teaching guide ranged from 21-47 with a mean score $\mathrm{M} \pm \mathrm{SD}$ (37.43 \pm 4.61$)$. Overall, this study found a statistical significant relationship among caregivers and thalassemia patients in six out of seven areas of knowledge that where assessed 
before and after distributing the teaching guide. Results of this study are parallel with other studies (Romeo, Di Gregorio et al. 1997, Yang, Chen et al. 2001, Heydarnejad and Hasanpour-Dehkordi 2008, Wong, George et al. 2011) that found the education of parents regarding thalassemia could have significant positive effect on increasing their knowledge.

As results revealed in our study, there were significant differences in the mean scores regarding nutritional status before and after teaching guide (paired $\mathrm{t}$ test $=12.49, \mathrm{p}=0.000$ ). This could be explained that nutritional information included in the guide had an influence on their knowledge score that will reflect on nutritional behavior of thalassemia patients later on during their daily life.

Findings of this present study show no significant difference among three independent groups of caregivers regarding overall attitude score of four categories included in the questionnaire $(\mathrm{F}$ ratio $=0.926 ; \mathrm{P}=0.398)$. Through examining of each attitude-item separately, one item (q25) was statistically significant among the independent groups ( $F$ ratio $=3.216, p=0.042$ ). This item "question 25: the family would not like others to know their affected children particularly if the family has a history for thalassemia". These findings are agreement with other studies (Zahed and BOU-DAMES 1997, Alkuraya and Kilani 2001, Zahed, Nabulsi et al. 2002, Kourorian, Azarkeivan et al. 2014, Basu 2015) who found that the majority of their study sample has a positive attitude regarding thalassemia.

In Syria Al-Zir 2009 reported that carriers may decide to undergo genetic testing and counseling to assess potential risks to their children, including prenatal testing, so we have given emphasis to health education. We believe that these measures will improve the quality of our life (Al-Zir 2009). Also in Syria, Murad 2014 reported in his study conducted among Syrian families to evaluate the prenatal diagnosis (PND) of $\beta$-thalassemia and sickle cell anemia results pointing to a successful future for PND of $\beta$-thalassemia and sickle cell anemia in Syria, using a rapid and accurate molecular method (Murad, Moassas et al. 2014). Also in Syria Kyriacou 2000 mentioned that their findings provide a sound foundation on which to base a preventive program for thalassemia and we believe that the data that we present will facilitate the improvement of medical services such as carrier screening, genetic counseling, and prenatal diagnosis. Furthermore a detailed knowledge of the molecular pathology of $\beta$-thalassemia will strongly improve the prenatal diagnosis services in Syria (Kyriacou, Quobaili et al. 2000).

Thalassemia was commonly seen by the community as a stigma for the patients and their families, who were often isolated (Bush, Mandel et al. 1998). This led most parents to be very secretive about the illness, and to avoid discussion of the subject with friends and relatives. The ill children were literally hidden away, did not attend school, and were not allowed to play with 
other children (Bush, Mandel et al. 1998, Caocci, Efficace et al. 2012). In this present study, stigmatization was significantly noticed among families caring for thalassemia patients. This result was congruent with Gharaibeh et al (Gharaibeh, Amarneh et al. 2009) and (Wong, George et al. 2011) who found that stigmatization had many risks on thalassemia patients such as decreasing the opportunity for social interactions with increasing the chance for isolation and its negative impact in increasing the psychological burden, especially on adolescent patients aged (13-18) years old.

Old children and adolescents with thalassemia major have difficulty to develop positive body image. Caregivers reported that $(40.8 \%)$ of their children were different from peers. Such feeling is reported by many authors (Angastiniotis and Hadjiminas 1981, Politis 1998, Angastiniotis 2002, Aydinok, Erermis et al. 2005, Abetz, Baladi et al. 2006, Cao and Kan 2013). Differences in their appearance as facial aspect, growth retardation and skin color made them the subject of sidelong looks and curiosity (Massaglia, Pozzan et al. 1986, Nagaraj, Umashree et al. 2011). In the present study, the common causes of such feeling were physical changes as growth retardation, facial changes, weakness and exercise intolerance, skin color and abdominal enlargement. Therefore, this study revealed that $(67.7 \%)$ of children and young adults were disturbed from these differences as reported by caregivers. The vast majority of thalassemia patients told their parents about negative feelings coming from others. The findings of this study were similar to Abetz et al. who reported that the impact of iron chelation therapy on patients with thalassemia is high (Abetz, Baladi et al. 2006, Payne, Rofail et al. 2008), and with Beratis 1993 who found that thalassemia patients demonstrated a significantly greater frequency of disturbed behavior towards relatives and friends than the control subjects (Beratis 1993).

The results of this study showed that vast majority of patients attach much importance to their appearance. It was also found that $(84.5 \%)$ of thalassemia patients have special interests and hobbies, but unfortunately $(55.9 \%)$ of the patients did not engage in group activities and play. This is because of exercise intolerance and feeling of shamed face as mentioned by them. Practicing sport was one of the burdens that affects patients with thalassemia, as thalassemia leads to low hemoglobin level, fatigue and general weakness (Politis, Di Palma et al. 1990, Bush, Mandel et al. 1998, Gharaibeh, Amarneh et al. 2009). In our research sample over quarter of thalassemia patients had enlarged spleen.

Dental caries is common among patient with thalassemia major (A1Wahadni, Taani et al. 2002, Schrier 2002). This level of disease may be explained on the basis of chronic nature of thalassemia. The higher caries prevalence can be also attributed to the poor oral hygiene, improper dietary habits, lack of dental knowledge, and poor motivation of these patients. In the 
present study, results revealed that about third of thalassemia patients infrequently or didn't perform oral hygiene respectively. The vast majority of the patients used toothpaste and brush to clean their teeth daily. Therefore, parents are more concerned with serious physical problems, paying lesser attention to the dental ailments, and only seek dental care when the child is in pain. This poor oral hygiene might be changed after giving teaching that emphasis to educate patients regarding the effects of thalassemia on teeth (Mehdizadeh, Mehdizadeh et al. 2008).

Results reveal statistical significant differences observed among three groups of thalassemic patients $(\mathrm{F}$ ratio $=6.46, \mathrm{p}=0.002)$ regarding serum ferritin, this is parallel with study conducted with Tuysuz (Senol, Tiftik et al. 2016, Tuysuz and Tayfun 2017) who found that the mean serum ferritin levels were significantly higher than the normal values and high ferritin level had a negative impact on HRQoL. The present study showed that the mean score of ferritin of adolescent group was higher than other groups. These findings are in agreement with previous study (Beratis 1993) that found rejection of treatment by adolescents and young adults is a recognized problem, whereas these findings are inconsistent with Safizadeh et al (Safizadeh and Farahmandinia 2012) who found no statistical differences in all aspect of quality of life with serum ferritin level.

In the present study, results revealed that 115 out of $238(48.3 \%)$ of the sample used Desferal subcutaneously, while $(51.7 \%)$ used oral chelation (Exjade). Through revising the 16 steps of administering Desferal S.C that were included in the teaching guide for both caregivers and patients, results reveal fourteen steps out of sixteen steps were improved by comparing the percentage of each before and after distributing the teaching guide. Statistical significant differences were found in performing all these steps before and after distributing the guide through comparing the overall performance (12.52 \pm 1.77 compared to $14.07 \pm 1.01$ ). (Paired t test $=11.477, p=0.000)$. The result is supported by a study carried by Abu- Salim who reported that skill score of administrating Desferal S.C was improved after a self- care program for children with thalassemia (Abu-Salim L 1999). In addition, chelation therapy indeed is difficult to accept because it is a daily reminder of one's own illness (Roy and Chatterjee 2007). It is usually performed in the evening when patients seek greater reassurance to be able to sleep peacefully. They may therefore complain of difficulty getting to sleep and resting well. There is no doubt that compliance rate can be improved by health education of both thalassemic patients and their caregivers. This fact is supported by numerous studies (Tsiantis, Anastasopoulos et al. 1990, Politis 1998, Atkin and Ahmad 2000, Hoch, Göbel et al. 2000, Angastiniotis 2002)

In this study, overall quality of life score ranged from 21.7 to 99.1 , with a mean score $\mathrm{M} \pm \mathrm{SD}(62.3 \pm 18.0$ compared to $67.5 \pm 16.4)$ of adolescent and 
adult group respectively. These results are comparable to those described by (Burckhardt and Anderson 2003, Mann-Jiles and Morris 2009, Kaheni, Yaghobian et al. 2013, Senol, Tiftik et al. 2016) who reported that QoL has low to moderate correlation with physical health status.

No statistical significant differences were noticed between the two age groups regarding 24 items of the quality of life. However, mean scores in physical health, psychological, social relationships and environmental domains insignificantly better in adult rather than adolescent groups ( $>0.05)$. Through comparing the overall quality of life scores between the two age groups by taking into account all four domains together, results also reveal no statistical significant differences between the adolescent and adult group. This might indicate that the adult group adapted relatively well physically and psychologically to the burden of chronic illness and treatments. . Findings of this study are in agreement with a study conducted by (Amani, Fathi et al. 2015) who reported that the quality of life among the patients with thalassemia in Ardabil was very good, and (Ali, Tarawah et al. 2015) who found in his study conducted in KSA that the quality of life in thalassemia patients was similar to the control group. Findings of this study are in agreement with Kaheni et al (Kaheni, Yaghobian et al. 2013), Gharaibeh et al (Gharaibeh and Gharaibeh 2012) and Cheuk et al (Cheuk, Mok et al. 2008) that stated better quality of life with the passage of time. On other hand, our findings were inconsistent with other studies (Cheuk, Mok et al. 2008, Ansari, Baghersalimi et al. 2014) reported that the QOL in all 6 dimensions was lower in patients compared to the controls.

Furthermore, the findings showed that patient's age was not associated with HRQoL scores. These findings were parallel with (Gharaibeh and Gharaibeh 2012, Haghpanah, Nasirabadi et al. 2013, Ali, Tarawah et al. 2015, Amani, Fathi et al. 2015) who found there were no significant differences between age and quality of life among their study sample. On other hand our findings were inconsistent with those reported by Dahlui and colleagues (Dahlui, Hishamshah et al. 2009) who found there were significant differences in quality of life according to age, quality of life was lowered as age increased and with Dhirar et al (Dhirar, Khandekar et al. 2016) who reported that the total quality of life scores were better for boys as compared to girls but the difference was not statically significant $(\mathrm{p}=0.131)$.

No statistical significant differences were found between the two treatment groups (subcutaneous vs oral) regarding 24 items of the quality of life. However, mean scores for each independent domain were better in subcutaneous rather than oral group, but these mean scores were statistically insignificant $(\mathrm{p}>0.05)$. Through comparing the overall quality of life scores between the two groups, results also reveal that no statistical significant differences were found between the subcutaneous and oral group respectively, 
with a mean score $\mathrm{M} \pm \mathrm{SD}(65.5 \pm 17.9$ compared to $62.1 \pm 16.0)$.

The findings of current study were in agreement with Torcharus et al. 2011 (Torcharus and Pankaew 2011), (Kaheni, Yaghobian et al. 2013), Trachtenberg et al (Trachtenberg, Mednick et al. 2012) and (Ali, Tarawah et al. 2015) who reported that is no statistical significant differences were found in their studies regarding QOL based on type of iron chelation therapy, and these findings are inconsistent with other studies Surapolchai et al 2010 (Surapolchai, Satayasai et al. 2010) and Tavorncharoensap et al 2010 (Thavorncharoensap, Torcharus et al. 2010) who found there was statistical significant differences in quality of life scores due to iron chelation therapy. On the other hand, Dhirar et al (Dhirar, Khandekar et al. 2016), Goulas et al (Goulas, Kourakli-Symeonidis et al. 2012) and Cheuk et al. (Cheuk, Mok et al. 2008), Osborne et al. (Osborne, Lourenço et al. 2007) reported that patients on Desferasiox (oral chelation) had significantly better self-related QoL than patients on other iron chelation regimen. Other studies also reported that the use of Desferal was a strong impediment of QoL, and effective oral chelation therapy might improve QoL (Ratip, Skuse et al. 1995, Caro, Ward et al. 2002, Kuo, Tsai et al. 2006, Thavorncharoensap, Torcharus et al. 2010, Ansari, Baghersalimi et al. 2014, Senol, Tiftik et al. 2016).

\section{Conclusion}

In this study we assessed the effects of knowledge, attitude and practice of thalassemia patients and their caregivers, on the patient's quality of life. Our findings highlighted that there was lack and scarcity of patients' knowledge and skills regarding the disease. There has been a remarkable improvement in both knowledge and performance after patients received the teaching guide. It is recommended to pay more attention in different aspects of life of these patients helping to improve their quality of life in all dimensions. Furthermore, it is necessary to improve patients' and their families' awareness regarding this disease. We also found that thalassemia and its complications have a negative impact on physical function, education, time off school, difference from friends/sibling, social interaction, playing and engaging in group activities and stigmatization. In addition, patients with thalassemia and their families often require support in many aspects, especially psychological support to prevent mental disorders.

Moreover, quality of life of patients in the four domains; physical, social, psychological and environmental health regarding to the patients' age was above average, also it was above average in physical, psychological and environmental domains regarding the patients' gender while it was less than average in social domain in females. The findings show there were differences between the four domains of quality of life regarding two types of iron chelation treatment (subcutaneous and oral), subcutaneous treatment was 
higher in all domains but the overall quality of life was higher also in subcutaneous but not statistically significant.

Thalassemia patients have many limitations in their daily life. Proper strategies are required for an effective prevention and treatment of this disease. Medical staff should focus on disease specific educational programs for patients with thalassemia about the entity and the process of the disease, its managements, self-care needs, importance of regular iron chelation therapy and its role in preventing or delaying complications. By improving these methods and giving much more social and psychological support, we could improve their quality of life.

\section{Acknowledgement}

The authors are grateful to acknowledge with extreme thanks the patients and their parents who participated and cooperated us in this study. Also we would like to thank Ministry of Health, the National Thalassemic center in Damascus and University of Gezira who supported us during this study.

\section{References:}

1. Abetz, L., et al. (2006). "The impact of iron overload and its treatment on quality of life: results from a literature review." Health and Quality of Life Outcomes 4(1): 73.

2. Al-Zir, K. N. (2009). "Prevention of hemoglobinopathies in Syria." Hemoglobin 33(sup1): S25-S27.

3. Al-Wahadni, A. M., et al. (2002). "Dental diseases in subjects with $\beta$ thalassemia major." Community dentistry and oral epidemiology 30(6): 418-422.

4. Al Hajeri, A. and S. Al Arrayed (2012). "Public awareness of beta thalassemia in Bahrain." Bahrain Medical Bulletin 34(1): 26-29.

5. Ali, S. S., et al. (2015). "Comprehensive patient care improves quality of life in transfusion dependent patients with $\beta$-thalassemia." Saudi medical journal 36(5): 575.

6. Alkuraya, F. S. and R. A. Kilani (2001). "Attitude of Saudi families affected with hemoglobinopathies towards prenatal screening and abortion and the influence of religious ruling (Fatwa)." Prenatal diagnosis 21(6): 448-451.

7. Amani, F., et al. (2015). "Quality of life among Ardabil patients with beta-thalassemia major."

8. Angastiniotis, M. (2002). "The adolescent thalassemic. The complicant rebel." Minerva pediatrica 54(6): 511-515.

9. Angastiniotis, M. and M. Hadjiminas (1981). "Prevention of thalassaemia in Cyprus." The Lancet 317(8216): 369-371. 
10. Ansari, S., et al. (2014). "Quality of life in patients with thalassemia major." Iranian journal of pediatric hematology and oncology 4(2): 57.

11. Atkin, K. and W. Ahmad (2000). "Pumping iron: compliance with chelation therapy among young people who have thalassaemia major." Sociology of Health \& Illness 22(4): 500-524.

12. Aydinok, Y., et al. (2005). "Psychosocial implications of thalassemia major." Pediatrics International 47(1): 84-89.

13. Ayoub, M. D., et al. (2013). "Quality of life among children with betathalassemia major treated in Western Saudi Arabia." Saudi Med J 34(12): 1281-1286.

14. Basu, M. (2015). "A study on knowledge, attitude and practice about thalassemia among general population in outpatient department at a Tertiary Care Hospital of Kolkata." J Preven Medic Holistic Health 1(1): 6-13.

15. Beratis, S. (1993). "Psychosocial status in pre-adolescent children with $\beta$-thalassaemia." Journal of psychosomatic research 37(3): 271-279.

16. Borgna-Pignatti, C. and M. R. Gamberini (2011). "Complications of thalassemia major and their treatment." Expert review of hematology 4(3): 353-366.

17. Burckhardt, C. S. and K. L. Anderson (2003). "The Quality of Life Scale (QOLS): reliability, validity, and utilization." Health and quality of life outcomes 1(1): 60 .

18. Bush, S., et al. (1998). "Future orientation and life expectations of adolescents and young adults with thalassemia major." Annals of the New York Academy of Sciences 850(1): 361-369.

19. Cao, A. and Y. W. Kan (2013). "The prevention of thalassemia." Cold Spring Harbor perspectives in medicine 3(2): a011775.

20. Caocci, G., et al. (2012). "Health related quality of life in Middle Eastern children with beta-thalassemia." BMC blood disorders 12(1): 6.

21. Capellini, M., et al. (2008). "Guidelines for the clinical management of thalassemia." Thalassaemia International Federation (TIF) April 2000 .

22. Caro, J. J., et al. (2002). "Impact of thalassemia major on patients and their families." Acta haematologica 107(3): 150-157.

23. Cheuk, D., et al. (2008). "Quality of life in patients with transfusiondependent thalassemia after hematopoietic SCT." Bone marrow transplantation 42(5): 319.

24. Cianciulli, P. (2008). "Treatment of iron overload in thalassemia." Pediatric endocrinology reviews: PER 6: 208-213.

25. Cohen, A. R. (2006). "New advances in iron chelation therapy." $\underline{\mathrm{ASH}}$ Education Program Book 2006(1): 42-47. 
26. Dahlui, M., et al. (2009). "Quality of life in transfusion-dependent thalassaemia patients on desferrioxamine treatment." Singapore medical journal 50(8): 794.

27. Dehkordi, A. H. and M. S. Heydarnejad (2008). Enhancement of parents' awareness about ß-thalassemia major disorder through two educational programs, Professional Medical Publications.

28. Dhirar, N., et al. (2016). "Thalassemia Major: how do we improve quality of life?" SpringerPlus 5(1): 1895.

29. El Dakhakhny, A. M., et al. (2011). "Quality of Life of School Age Thalassemic Children at Zagazig City Amal M El Dakhakhny, Mervat A Hesham, Samah E Mohamed, Fawzia N Mohammad." Journal of American Science 7(1).

30. Elalfy, M. S., et al. (2014). "Quality of life of Egyptian b-thalassemia major children and adolescents." The Egyptian Journal of Haematology 39(4): 222.

31. Gharaibeh, H., et al. (2009). "The psychological burden of patients with beta thalassemia major in Syria." Pediatrics international 51(5): 630-636.

32. Gharaibeh, H. and M. Gharaibeh (2012). "Factors influencing healthrelated quality of life of thalassaemic Jordanian children." Child: care, health and development 38(2): 211-218.

33. Gharaibeh, H. and F. Mater (2009). "Young Syrian adults' knowledge, perceptions and attitudes to premarital testing." International nursing review 56(4): 450-455.

34. Goulas, V., et al. (2012). "Comparative effects of three iron chelation therapies on the quality of life of greek patients with homozygous transfusion-dependent Beta-thalassemia." ISRN hematology 2012.

35. Haghpanah, S., et al. (2013). "Quality of life among Iranian patients with beta-thalassemia major using the SF-36 questionnaire." Sao Paulo Medical Journal 131(3): 166-172.

36. Hanl, A. M., et al. (1992). "Thalassemia in the outpatient department of the Yangon Children's Hospital in Myanmar: cost analysis of the day-care-room services for thalassemia." Medicine 3(2): 3,183.

37. Hatami, G. and N. Motamed (2014). "Life Satisfaction in children and adolescents with beta thalassemia major in southwest Iran." Electronic physician 6(1): 759.

38. Heydarnejad, M. and A. Hasanpour-Dehkordi (2008). "Effect of booklet and combined method on parents' awareness of children with $\beta$-thalassemia major disorder." Journal of the Pakistan Medical Association 58(9).

39. Hoch, C., et al. (2000). "Psychosocial support of patients with homozygous beta-thalassaemia." Klinische Padiatrie 212(4): 216-219. 
40. Ishaq, F., et al. (2012). "Awareness Among Parents of $\beta \beta$-Thalassemia Major Patients, Regarding Prenatal Diagnosis and Premarital Screening." Journal of the College of Physicians and Surgeons Pakistan 22(4): 218-221.

41. Jafari, H., et al. (2008). "Quality of life in thalassemia major: reliability and validity of the Persian version of the SF-36 questionnaire." Journal of postgraduate medicine 54(4): 273.

42. Jantan, A. W., et al. (2011). "Thalassaemia: a study on the perception of patients and family members." Medical Journal of Malaysia 66(4): 326-334.

43. Kaheni, S., et al. (2013). "Quality of life in children with $\beta$-thalassemia major at center for special diseases." Iranian journal of pediatric hematology and oncology 3(3): 108.

44. Khurana, A., et al. (2006). "Psychosocial burden in thalassemia." The Indian Journal of Pediatrics 73(10): 877-880.

45. Kourorian, Z., et al. (2014). The effect of knowledge, attitude and practice on the function of thalassemic patients.

46. Kuo, H.-T., et al. (2006). "Pilot study on the "quality of life" as reflected by psychosocial adjustment of children with thalassemia major undergoing iron-chelating treatment in western Taiwan." Hemoglobin 30(2): 291-299.

47. Kyriacou, K., et al. (2000). "Molecular characterization of $\beta$ thalassemia in Syria." Hemoglobin 24(1): 1-13.

48. Liem, R. I., et al. (2011). "THE IMPACT OF THALASSEMIA ON SOUTHEAST ASIAN AND ASIAN INDIAN FAMILIES IN THE UNITED STATES: AQualitative STUDY." childhood 11: 12.

49. Mann-Jiles, V. and D. L. Morris (2009). "Quality of life of adult patients with sickle cell disease." Journal of the American Association of Nurse Practitioners 21(6): 340-349.

50. Massaglia, P., et al. (1986). "Psychological aspects of Thalassemia." La Pediatria medica e chirurgica: Medical and surgical pediatrics 8(1): 27-31.

51. Mehdizadeh, M., et al. (2008). "Orodental complications in patients with major beta-thalassemia." Dent Res J 5(1): 17-20.

52. Milat, F., et al. (2012). "A case of hypophosphatemic osteomalacia secondary to deferasirox therapy." Journal of Bone and Mineral Research 27(1): 219-222.

53. Miri-Moghaddam, E., et al. (2011). "Molecular basis and prenatal diagnosis of $\beta$-thalassemia among Balouch population in Iran." Prenatal diagnosis 31(8): 788-791.

54. Mrayyan, M. T., et al. "Jordanian Families' Attitudes toward Thalassaemia and Genetic Counseling." 
55. Muncie Jr, H. L. and J. Campbell (2009). "Alpha and beta thalassemia." American family physician 80(4): 339-344.

56. Murad, H., et al. (2014). "Prenatal Molecular Diagnosis of $\beta$ Thalassemia and Sickle Cell Anemia in the Syrian Population." Hemoglobin 38(6): 390-393.

57. Nagaraj, T., et al. (2011). "[Beta] Thalassemia major: A case report." Journal of International Oral Health 3(5): 67.

58. Olivieri, N. F., et al. (1994). "Survival in medically treated patients with homozygous $\beta$-thalassemia." New England Journal of Medicine 331(9): 574-578.

59. Osborne, R. H., et al. (2007). "Quality of life related to oral versus subcutaneous iron chelation: a time trade-off study." Value in health 10(6): 451-456.

60. Othman, H. and M. Saadat (2009). "Prevalence of consanguineous marriages in Syria." Journal of biosocial science 41(5): 685-692.

61. Pakbaz, Z., et al. (2005). "A simple model to assess and improve adherence to iron chelation therapy with deferoxamine in patients with thalassemia." Annals of the New York Academy of Sciences 1054(1): 486-491.

62. Pakbaz, Z., et al. (2005). "A simple model to assess and improve adherence to iron chelation therapy with deferoxamine in patients with thalassemia." Annals of the New York Academy of Sciences 1054: 486-491.

63. Patricia Ault, R. (2009). "Understanding iron overload: screening, monitoring, and caring for patients with transfusion-dependent anemias." Clinical journal of oncology nursing 13(5): 511.

64. Payne, K. A., et al. (2008). "Iron chelation therapy: clinical effectiveness, economic burden and quality of life in patients with iron overload." Advances in therapy 25(8): 725.

65. Pennell, D., et al. (2011). "On improvement in ejection fraction with iron chelation in thalassemia major and the risk of future heart failure." Journal of Cardiovascular Magnetic Resonance 13(1): 45.

66. Perisano, C., et al. (2012). "Physiopathology of bone modifications inthalassemia." Anemia 2012.

67. Politis, C. (1998). "The psychosocial impact of chronic illness." Annals of the New York Academy of Sciences 850(1): 349-354.

68. Politis, C., et al. (1990). "Social integration of the older thalassaemic patient." Archives of disease in Childhood 65(9): 984-986.

69. Prasomsuk, S., et al. (2007). "Lived experiences of mothers caring for children with thalassemia major in Thailand." Journal for Specialists in Pediatric Nursing 12(1): 13-23. 
70. Ratip, S., et al. (1995). "Psychosocial and clinical burden of thalassaemia intermedia and its implications for prenatal diagnosis." Archives of disease in childhood 72(5): 408-412.

71. Romeo, M. A., et al. (1997). "Persistence of Mediterranean anaemia in Sicily." Journal of medical genetics 34(10): 869-870.

72. Roy, T. and S. C. Chatterjee (2007). "The experiences of adolescents with thalassemia in West Bengal, India." Qualitative Health Research 17(1): 85-93.

73. Safizadeh, H. and Z. Farahmandinia (2012). "Quality of life in patients with thalassemia major and intermedia in kerman-iran (IR)." Mediterranean journal of hematology and infectious diseases 4(1).

74. Scalone, L., et al. (2008). "Costs, quality of life, treatment satisfaction and compliance in patients with $\beta$-thalassemia major undergoing iron chelation therapy: the ITHACA study." Current medical research and opinion 24(7): 1905-1917.

75. Schrier, S. L. (2002). "Pathophysiology of thalassemia." Current opinion in hematology 9(2): 123-126.

76. Schrier, S. L. and E. Angelucci (2005). "New strategies in the treatment of the thalassemias." Annu. Rev. Med. 56: 157-171.

77. Senol, S. P., et al. (2016). "Quality of life, clinical effectiveness, and satisfaction in patients with beta thalassemia major and sickle cell anemia receiving deferasirox chelation therapy." Journal of basic and clinical pharmacy 7(2): 49.

78. Seyam, S. and A. Assemi (2010). "Study of the knowledge in guilan university students about thalassemia." Journal of Urmia Nursing And Midwifery Faculty 8(3): 0-0.

79. Seyedifar, M., et al. (2016). "Health-related quality of life and health utility values in beta thalassemia major patients receiving different types of iron chelators in Iran." International journal of hematologyoncology and stem cell research 10(4): 224.

80. Shaligram, D., et al. (2007). "Psychological problems and quality of life in children with thalassemia." The Indian Journal of Pediatrics 74(8): 727-730.

81. Shrivastava, K., et al. (2011). "Knowledge, attitude and practice study of beta-thalassemia in rural bengal." Genetic clinics 4(4): 13-15.

82. Surapolchai, P., et al. (2010). "Biopsychosocial predictors of healthrelated quality of life in children with thalassemia in Thammasat University Hospital." J Med Assoc Thai 93(Suppl 7): 65-75.

83. Telfer, P., et al. (2005). "Quality of life in thalassemia." Annals of the New York Academy of Sciences 1054(1): 273-282. 
84. Thavorncharoensap, M., et al. (2010). "Factors affecting health-related quality of life in Thai children with thalassemia." BMC Hematology 10(1): 1 .

85. Torcharus, K. and T. Pankaew (2011). "Health-related quality of life in Thai thalassemic children treated with iron chelation." Southeast Asian Journal of Tropical Medicine and Public Health 42(4): 951.

86. Touma, H., et al. (2013). Clinical Effectiveness of Iron Chelation Therapies in Syrian Patients with $\beta$ Thalassemia Major: Suboptimal Clinical Outcomes and High Prevalence of Iron Overload, Am Soc Hematology.

87. Trachtenberg, F. L., et al. (2012). "Beliefs about chelation among thalassemia patients." Health and quality of life outcomes 10(1): 148.

88. Tsiantis, J., et al. (1990). "A multi-level intervention approach for care of HIV-positive haemophiliac and thalassaemic patients and their families." AIDS care 2(3): 253-266.

89. Tuysuz, G. and F. Tayfun (2017). "Health-related Quality of Life and its Predictors Among Transfusion-dependent Thalassemia Patients." Journal of pediatric hematology/oncology 39(5): 332-336.

90. Vichinsky, E., et al. (2008). "Standards of care guidelines for thalassemia." Children's Hospital \& Research Center Oakland, USA.

91. Wong, L. P., et al. (2011). "Public perceptions and attitudes toward thalassaemia: Influencing factors in a multi-racial population." BMC Public Health 11(1): 193.

92. Wonke, B. (2001). Clinical management of $\beta$-thalassemia major. Seminars in hematology, Elsevier.

93. Yang, H., et al. (2001). "Illness knowledge, social support and self care behavior in adolescents with beta-thalassemia major." $\mathrm{Hu}$ li yan jiu= Nursing research 9(2): 114-124.

94. Zahed, L. and J. BOU-DAMES (1997). "ACCEPTANCE OF FIRSTTRIMESTER PRENATAL DIAGNOSIS FOR THE HAEMOGLOBINOPATHIES IN LEBANON." Prenatal diagnosis 17(5): 423-428.

95. Zahed, L., et al. (2002). "Attitudes towards prenatal diagnosis and termination of pregnancy among health professionals in Lebanon." Prenatal diagnosis 22(10): 880-886.

96. Zani, B., et al. (1995). "Psychosocial aspects of chronic illness in adolescents with thalassaemia major." Journal of adolescence 18(4): 387-402. 